\title{
High volatility, thick tails and extreme value theory in value-at-risk estimation
}

\author{
Ramazan Gençay $^{a, *}$, Faruk Selçuk ${ }^{b, 1}$, Abdurrahman Ulugülyağci ${ }^{b}$ \\ ${ }^{a}$ Department of Economics, Carleton University, 1125 Colonel By Drive, Ottawa, Ont., Canada K1S $5 B 6$ \\ b Department of Economics, Bilkent University, Bilkent 06533, Ankara, Turkey
}

Received 1 August 2002; received in revised form 3 May 2003; accepted 29 July 2003

\begin{abstract}
In this paper, the performance of the extreme value theory in value-at-risk calculations is compared to the performances of other well-known modeling techniques, such as GARCH, variance-covariance (Var-Cov) method and historical simulation in a volatile stock market. The models studied can be classified into two groups. The first group consists of $\operatorname{GARCH}(1,1)$ and GARCH $(1,1)-t$ models which yield highly volatile quantile forecasts. The other group, consisting of historical simulation, Var-Cov approach, adaptive generalized Pareto distribution (GPD) and nonadaptive GPD models, leads to more stable quantile forecasts. The quantile forecasts of $\operatorname{GARCH}(1,1)$ models are excessively volatile relative to the GPD quantile forecasts. This makes the GPD model be a robust quantile forecasting tool which is practical to implement and regulate for VaR measurements.
\end{abstract}

(c) 2003 Elsevier B.V. All rights reserved.

JEL classification: $\mathrm{G} 0 ; \mathrm{G} 1 ; \mathrm{C} 1$

Keywords: Value-at-risk; Financial risk management; Extreme value theory

\section{Introduction}

The common lesson from financial disasters is that billions of dollars can be lost because of poor supervision and management of financial risks. The value-at-risk (VaR) was developed in response to financial disasters of the 1990s and obtained an increasingly important role in market risk management. The VaR summarizes the worst loss over a target horizon with a given level of confidence. It is a popular approach because it provides a single quantity

\footnotetext{
* Corresponding author. Fax: +1-208-693-7012. E-mail addresses: gencay@carleton.ca (R. Gençay), faruk@bilkent.edu.tr (F. Selçuk).

1 Tel.: +90-312-290-2074; fax: +90-208-694-3196.
}

that summarizes the overall market risk faced by an institution or an individual investor. ${ }^{2}$

In a VaR context, precise prediction of the probability of an extreme movement in the value of a portfolio is essential for both risk management and regulatory purposes. By their very nature, extreme movements are related to the tails of the distribution of the underlying data generating process. Several tail studies, after the pioneering work by Mandelbrot (1963a,b), indicate that most financial time series are fat-tailed. ${ }^{3}$ Although

\footnotetext{
${ }^{2}$ See Dowd (1998), Jorion (1997) and Duffie and Pan (1997) for more details on the VaR methodology. For the regulatory roots of the VaR, see Basel (1996).

${ }^{3}$ See, for example, Dacorogna et al. (2001a,b), Hauksson et al. (2001), Müller et al. (1998), Pictet et al. (1998), Danielsson and de Vries (1997), Ghose and Kroner (1995), Loretan and Phillips (1994), Hols and de Vries (1991), Koedijk et al.
} 
these findings necessitate a definition of what is meant by a fat-tailed distribution, there is no unique definition of fat-tailness (heavy-tailness) of a distribution in the literature. ${ }^{4}$ In this study, we consider a distribution to be fat-tailed if a power decay of the density function is observed in the tails. Accordingly, an exponential decay or a finite endpoint at the tail (the density reaching zero before a finite quantile) is treated as thin-tailed. ${ }^{5}$

In order to model fat-tailed distributions, the lognormal distribution, generalized error distribution, and mixtures of normal distributions are suggested in many studies. However, these distributions are thintailed according to our definition since the tails of these distributions decay exponentially, although they have excess kurtosis over the normal distribution. In some practical applications, these distributions may fit the empirical distributions up to moderate quantiles but their fit deteriorates rapidly at high quantiles (at extremes).

An important issue in modeling the tails is the finiteness of the variance of the underlying distribution. The finiteness of the variance is related to the thickness of the tails and the evidence of heavy tails in financial asset returns is plentiful. In his seminal work, Mandelbrot (1963a,b) advanced the hypothesis of a stable distribution on the basis of an observed invariance of the return distribution across different frequencies and apparent heavy tails in return distributions. The issue is that while the normal distribution provides a good approximation to the center of the return distribution for monthly (and lower) data frequencies, there is strong deviation from normality for frequencies higher than monthly frequency. This implies that there is a higher probability of extreme values than for a normal distribution. ${ }^{6}$ Mandelbrot (1963a,b) provided empirical evidence that the stable Levy distributions are natural candidates for return

\footnotetext{
(1990), Boothe and Glassman (1987), Levich (1985) and Mussa (1979).

${ }^{4}$ See Embrechts et al. (1997, Chapters 2 and 8) for a detailed discussion.

5 Although the fourth moment of an empirical distribution (sample kurtosis) is sometimes used to decide on whether an empirical distribution is heavy-tailed or not, this measure might be misleading. For example, the uniform distribution has excess kurtosis over the normal distribution but it is thin-tailed according to our definition.

6 This indicates that the fourth moment of the return distribution is larger than expected from a normal distribution.
}

distributions. For excessively fat-tailed random variables whose second moment does not exist, the standard central limit theorem no longer applies, however, the sum of such variables converge to Levy distribution within a generalized central limit theorem. Later studies, ${ }^{7}$ however, demonstrated that the return behavior is much more complicated, and follows a power law, which is not compatible with the Levy distribution.

Instead of forcing a single distribution for the entire sample, it is possible to investigate only the tails of the sample distribution using limit laws, if only the tails are important for practical purposes. Furthermore, the parametric modeling of the tails is convenient for the extrapolation of probability assignments to the quantiles even higher than the most extreme observation in the sample. One such approach is the extreme value theory (EVT) which provides a formal framework to study the tail behavior of the fat-tailed distributions.

The EVT stemming from statistics has found many applications in structural engineering, oceanography, hydrology, pollution studies, meteorology, material strength, highway traffic and many others. ${ }^{8}$ The link between the EVT and risk management is that EVT methods fit extreme quantiles better than the conventional approaches for heavy-tailed data. ${ }^{9}$ The EVT approach is also a convenient framework for the separate treatment of the tails of a distribution which allows for asymmetry. Considering the fact that most financial return series are asymmetric (Levich, 1985; Mussa, 1979), the EVT approach is advantageous over models which assume symmetric distributions such as $t$-distributions, normal distributions, ARCH, GARCH-like distributions except E-GARCH which allows for asymmetry (Nelson, 1991). Our findings indicate that the performance of conditional risk management strategies, such as ARCH and GARCH, is relatively poor as compared to unconditional approaches.

The paper is organized as follows. The EVT and VaR estimation are introduced in Sections 2 and 3.

\footnotetext{
7 See Koedijk et al. (1990), Mantegna and Stanley (1995), Lux (1996), Müller et al. (1998) and Pictet et al. (1998).

${ }^{8}$ For an in-depth coverage of EVT and its applications in finance and insurance, see Embrechts et al. (1997), McNeil (1998), Reiss and Thomas (1997) and Teugels and Vynckier (1996).

${ }^{9}$ See Embrechts et al. (1999) and Embrechts (2000a) for the efficiency of EVT as a risk management tool.
} 
Empirical results from a volatile market are presented in Section 4. We conclude afterwards.

\section{Extreme value theory}

From the practitioners' point of view, one of the most interesting questions that tail studies can answer is what are the extreme movements that can be expected in financial markets? Have we already seen the largest ones or are we going to experience even larger movements? Are there theoretical processes that can model the type of fat tails that come out of our empirical analysis? Answers to such questions are essential for sound risk management of financial exposures. It turns out that we can answer these questions within the framework of the EVT. Once we know the tail index, we can extend the analysis outside the sample to consider possible extreme movements that have not yet been observed historically. This can be achieved by computation of the quantiles with exceedance probabilities.

EVT is a powerful and yet fairly robust framework to study the tail behavior of a distribution. Even though EVT has previously found large applicability in climatology and hydrology, there have also been a number of extreme value studies in the finance literature in recent years. de Haan et al. (1994) study the quantile estimation using the EVT. Reiss and Thomas (1997) is an early comprehensive collection of statistical analysis of extreme values with applications to insurance and finance, among other fields. McNeil (1997, 1998) studies the estimation of the tails of loss severity distributions and the estimation of the quantile risk measures for financial time series using EVT. Embrechts et al. (1999) overview the EVT as a risk management tool. Müller et al. (1998) and Pictet et al. (1998) study the probability of exceedances for the foreign exchange rates and compare them with the GARCH and HARCH models. Embrechts (1999, 2000a) studies the potentials and limitations of the EVT. McNeil (1999) provides an extensive overview of the EVT for risk managers. McNeil and Frey (2000) study the estimation of tail-related risk measures for heteroskedastic financial time series. Embrechts et al. (1997), Embrechts (2000b) and Reiss and Thomas (1997) are comprehensive sources of the EVT to the finance and insurance literature.

\section{Value-at-risk}

Let $r_{t}=\log \left(p_{t} / p_{t-1}\right)$ be the returns at time $t$ where $p_{t}$ is the price of an asset (or portfolio) at time $t$. The $\operatorname{VaR}_{t}(\alpha)$ at the $(1-\alpha)$ percentile is defined by

$\operatorname{Pr}\left(r_{t} \leq \operatorname{VaR}_{t}(\alpha)\right)=\alpha$,

which calculates the probability that returns at time $t$ will be less than (or equal to) $\operatorname{VaR}_{t}(\alpha), \alpha$ percent of the time. ${ }^{10}$ The VaR is the maximum potential increase in value of a portfolio given the specifications of normal market conditions, time horizon and a level of statistical confidence. The VaR's popularity originates from the aggregation of several components of risk at firm and market levels into a single number.

The acceptance and usage of VaR has been spreading rapidly since its inception in the early 1990s. The $\mathrm{VaR}$ is supported by the group of 10 banks, the group of 30, the Bank for International Settlements, and the European Union. The limitations of the VaR are that it may lead to a wide variety of results under a wide variety of assumptions and methods; focuses on a single somewhat arbitrary point; explicitly does not address exposure in extreme market conditions and it is a statistical measure, not a managerial/economic one. $^{11}$

The methods used for VaR can be grouped under the parametric and nonparametric approaches. In this paper, we study the VaR estimation with EVT which is a parametric approach. The advantage of the EVT is that it focuses on the tails of the sample distribution when only the tails are important for practical purposes. Since fitting a single distribution to the entire sample imposes too much structure and our need here is the tails, we adopt the EVT framework which is what is needed to calculate the VaR. We compare the VaR calculations with EVT and its performance to the variance-covariance (Var-Cov) method (parametric, unconditional volatility), historical simulation (nonparametric, unconditional volatility), GARCH

\footnotetext{
${ }^{10}$ A typical value of $\alpha$ is 5 or $1 \%$.

11 There is a growing consensus among both academicians and practitioners that the VaR as a measure of risk has serious deficiencies. See the special issue of Journal of Banking and Finance 26 (7) (2002) on "Statistical and Computational Problems in Risk Management: VaR and Beyond VaR".
} 
$(1,1)-t$ and $\operatorname{GARCH}(1,1)$ with normally distributed innovations (parametric, conditional volatility).

The Var-Cov method is the simplest approach among the various models used to estimate the VaR. Let the sample of observations be denoted by $r_{t}$, $t=1,2, \ldots, n$, where $n$ is the sample size. Let us assume that $r_{t}$ follows a martingale process with $r_{t}=\mu_{t}+\epsilon_{t}$, where $\epsilon$ has a distribution function $F$ with zero mean and variance, $\sigma_{t}^{2}$. The $\mathrm{VaR}$ in this case can be calculated as

$\operatorname{VaR}_{t}(\alpha)=\hat{\mu}_{t}+F^{-1}(\alpha) \hat{\sigma}_{t}$,

where $F^{-1}(\alpha)$ is the $q$ th quantile $(q=1-\alpha)$ value of the unknown distribution function $F$. An estimate of $\mu_{t}$ and $\sigma_{t}^{2}$ can be obtained from the sample mean and the sample variance. Although sample variance as an estimator of the standard deviation in Var-Cov approach is simple, it has drawbacks at high quantiles of a fat-tailed empirical distribution. The quantile estimates of the Var-Cov method for the right tail (left tail) are biased downwards (upwards) for high quantiles of a fat-tailed empirical distribution. Therefore, the risk is underestimated with this approach. Another drawback of this method is that it is not appropriate for asymmetric distributions. Despite these drawbacks, this approach is commonly used for calculating the VaR from holding a certain portfolio, since the VaR is additive when it is based on sample variance under the normality assumption.

Instead of the sample variance, the standard deviation in Eq. (2) can be estimated by a statistical model. Since financial time series exhibit volatility clustering, the autoregressive conditional heteroscedasticity (ARCH) (Engle, 1982) and the generalized autoregressive conditional heteroscedasticity (GARCH) (Bollerslev, 1982) are popular models in practice. Among other studies, Danielsson and Moritomo (2000) and Danielsson and de Vries (2000) show that these conditional volatility models with frequent parameter updates produce volatile estimates, and are not well suited for analyzing large risks. Our findings provide further evidence that the performance of conditional risk management strategies, such as ARCH and GARCH, is relatively poor as compared to unconditional approaches. The performance of these conditional models worsens as one moves further in the tail of the losses.

\section{Empirical findings}

A volatile market provides a suitable environment to study the relative performance of competing $\mathrm{VaR}$ modeling approaches. In this regard, the Turkish economy is a good candidate. ${ }^{12}$ The Istanbul Stock Exchange was formally inaugurated at the end of 1985 . Following the capital account liberalization in 1989, foreign investors were allowed to purchase and sell all types of securities and repatriate the proceeds. The total market value of all traded companies was only US\$ 938 million in 1986 and reached a record level of US\$ 114.3 billion in 1999. Following a major financial crisis in February 2001, the total market value of all listed companies went down to 33.1 billion by November 2001. The daily average trade volume also decreased from the record level US\$ 740 million in 2000 to US\$ 336 million in November 2001. Figs. 1 and 2 clearly indicate the high volatility and thick-tail nature of the Istanbul Stock Exchange Index (ISE-100), making it a natural platform to study EVT in financial markets.

\subsection{Data analysis}

The data set is the daily closings of the Istanbul Stock Exchange (ISE-100) Index from 2 November 1987 to 8 June 2001 . The index value is normalized to 1 at 1 January 1986 and there are 3383 observations in the data set. The daily returns are defined by $r_{t}=\log \left(p_{t} / p_{t-1}\right)$, where $p_{t}$ denotes the value of the index at day $t$. In the top panel of Fig. 1 the level of the ISE-100 Index is presented. The corresponding daily returns are displayed in the bottom panel of Fig. 1. The average daily return is $0.22 \%$ which implies approximately $77 \%$ annual return (260 business days). ${ }^{13}$ This is not surprising as the economy is a high inflation economy. However, $3.27 \%$ daily standard deviation indicates a highly volatile environment. Indeed, extremely high daily returns (as high as $30.5 \%$ ) or daily losses (as low as $-20 \%$ ) are observed during the sample period. Also from a foreign investor's point of view, ISE-100 exhibits a wide degree of fluctuations which is reflected in its US dollar value. In US dollar

\footnotetext{
12 For an overview of the Turkish economy in recent years, see Ertuğrul and Selçuk (2001). Gençay and Selçuk (2001) study the recent crises episode in the Turkish economy from the risk management point of view.

${ }^{13} 1.0022^{260}=1.771$.
} 

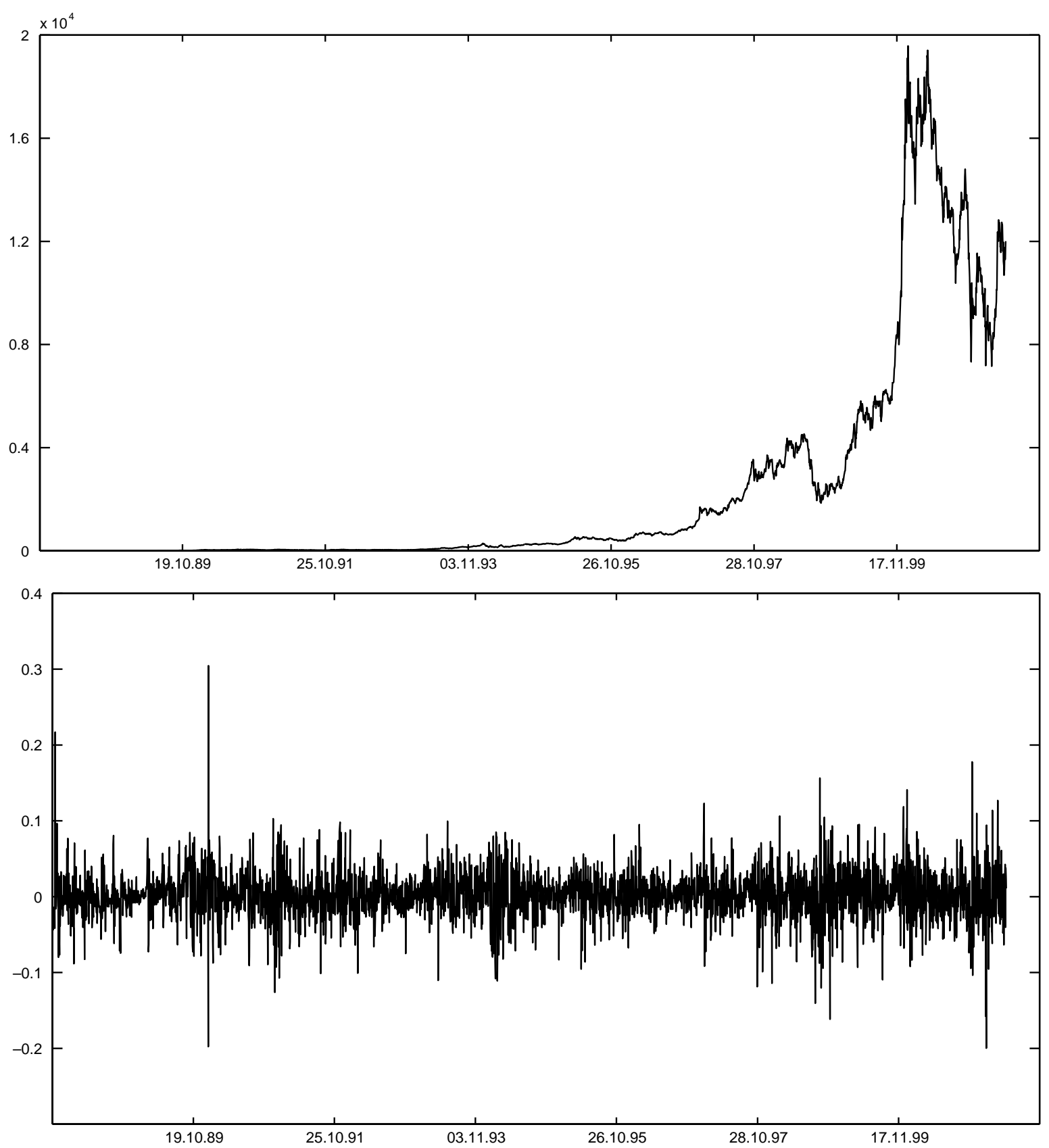

Fig. 1. Top: daily ISE-100 Index from 2 November 1987 to 8 June $2001(1$ January $1986=1)$. The horizontal axis corresponds to time while the vertical axis displays the value of the index. The last closing value of the index is 12138.26 , implying a daily return of $0.22 \%$ (geometric mean) on the average. Bottom: ISE-100 returns calculated by $r_{t}=\log \left(p_{t} / p_{t-1}\right)$, where $p_{t}$ is the value of the index at $t$. Notice that there is volatility clustering in the market. 

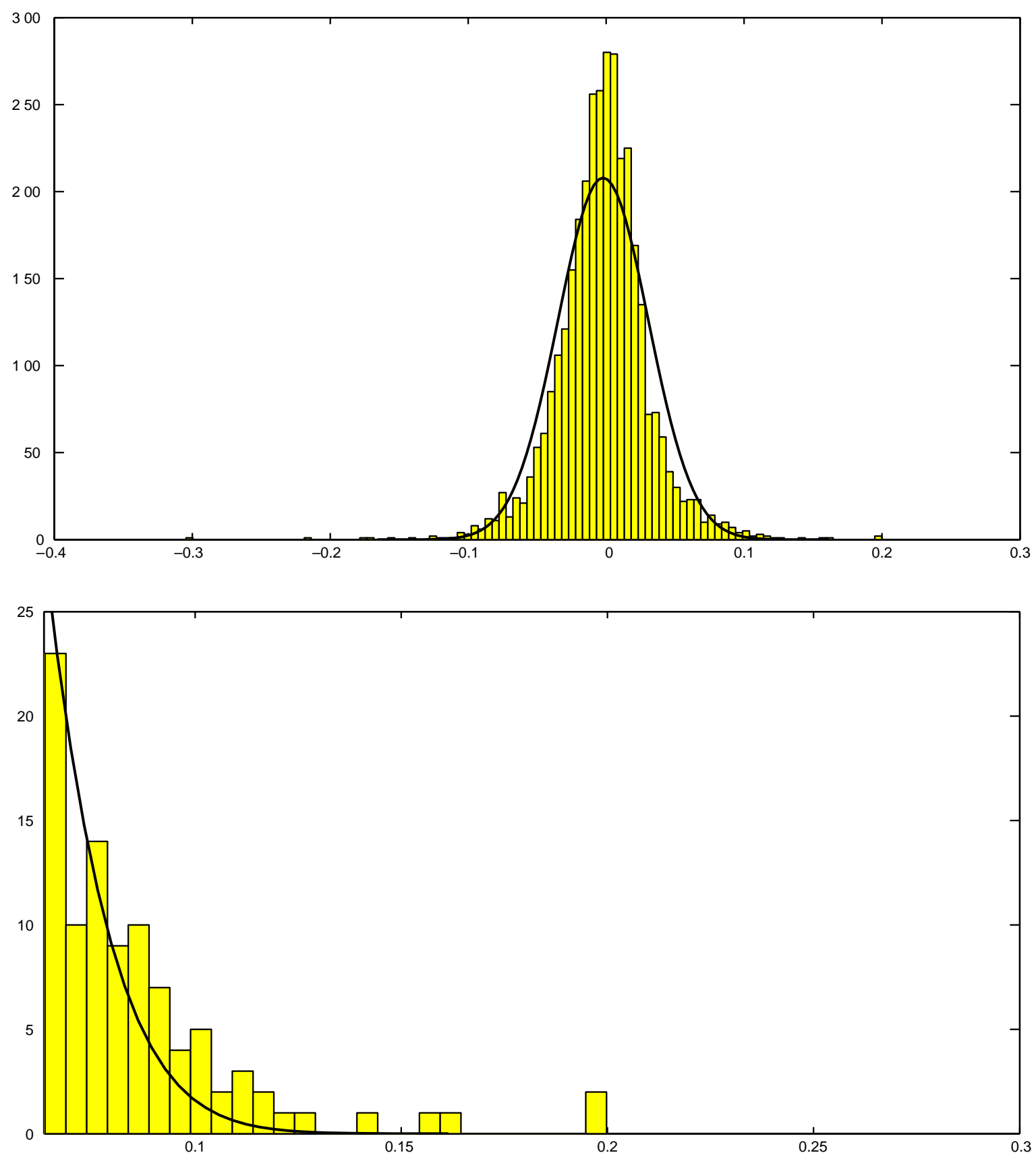

Fig. 2. Histogram of daily negative returns (losses) and the best fitted normal distribution, $N(\hat{\mu}, \hat{\sigma})$. Estimated parameters are $\hat{\mu}=-0.0022$ and $\hat{\sigma}=0.0327$. The right tail region (values over $\hat{\mu}+2 \hat{\sigma}=0.0633$ ) is zoomed in the lower panel which indicates a heavy tail. 
terms $(1986=100)$, the ISE-100 reached a record level of 1654 at the end of the year 1999 and dropped down to 378 in November 2001.

The sample skewness and kurtosis are 0.18 and 8.21, respectively. Although there is no significant skewness, there is excess kurtosis. In the framework of this paper, the fat-tailness may not be based on a normality test. Normality tests, such as the Bera and Jarque (1981) normality test, based on sample skewness and sample kurtosis, may not be appropriate since rejecting normality due to a significant skewness or a significant excess kurtosis does not necessarily imply fat-tailness. For instance, a distribution may be skewed and thin-tailed or the empirical distribution may have excess kurtosis over normal distribution with thin-tails. The first 300 autocorrelations and partial autocorrelations of squared returns are statistically significant at several lags. This indicates volatility clustering and a GARCH type modeling should be considered in VaR estimations.

Other important tools for the examination of fattailness in the data are the sample histogram, QQ (quantile-quantile) plot and the mean excess function. ${ }^{14}$ The sample histogram of negative returns (returns multiplied with -1 ) is presented in Fig. 2. Extreme value analysis works with the right tail of the distribution. Hence, we work with negative return distribution where the right tail corresponds to losses. ${ }^{15}$ Fig. 2 indicates that extreme realizations are more likely than a normal distribution would imply. The mean excess plot in the top-left panel of Fig. 3 indicates a heavy right tail for the loss distribution. QQ plot gives some idea about the underlying distribution of a sample. Specifically, the quantiles of an empirical distribution are plotted against the quantiles of a hypothesized distribution. If the sample comes from the hypothesized distribution or a linear transformation of the hypothesized distribution, the QQ plot should be linear. In extreme value analysis and generalized Pareto models, the unknown shape parameter of the distribution can be replaced by an estimate as suggested by Reiss and Thomas (1997, p. 66). If there is a strong deviation from a straight line, then either the assumed shape parameter is wrong or the model

\footnotetext{
14 See Reiss and Thomas (1997, Chapters 1 and 2) for some empirical tools for representing data and to check the validity of the parametric modeling.

15 Hereafter, we will refer to negative returns as losses.
}

selection is not accurate. In our case, the QQ plot of losses in the top-right panel of Fig. 3 provides further evidence for fat-tailness. The losses over a threshold are plotted with respect to generalized Pareto distribution (GPD) with an assumed shape parameter 0.20 . The plot clearly shows that the left tail of the distributions over the threshold value 0.08 is well approximated by GPD.

The Hill plot is used to calculate the shape parameter $\xi=1 / \alpha$, where $\alpha$ is the tail index. The shape parameter $\xi$ is informative regarding the limiting distribution of maxima. If $\xi=0, \xi>0$ or $\xi<0$, this indicates an exponentially decaying, power-decaying, or finite-tail distributions in the limit, respectively. The critical aspect of the Hill estimator is the choice of the number of upper order statistics. The Hill plot of losses is displayed in the bottom panel of Fig. 3. The stable portion of this figure implies a tail index estimate between 0.20 and 0.25 . Therefore, the Hill estimator indicates a power-decaying tail with an exponent which varies between 4 and 5. This means that if the probability of observing a return greater than $r$ is $p$ then the probability of observing a loss greater than $k r$ is in between $k^{-4} p$ and $k^{-5} p$.

\subsection{Relative performance}

We consider six different models for the one period ahead loss predictions at different tail quantiles. These models are Var-Cov approach, historical simulation, $\operatorname{GARCH}(1,1), \operatorname{GARCH}(1,1)-t$, adaptive GPD and nonadaptive GPD models.

For the first five models, we adopt a sliding window approach with three different window sizes for 500, 1000, and 2000 days. ${ }^{16}$ For instance, the window is placed between first and 1000th observations for a window size of 1000 days and a given quantile is forecasted for the 1001st day. Next, the window is slided one step forward to forecast quantiles for 1002nd, 1003rd, ..., 3382nd days. The motivation behind the sliding window technique is to capture dynamic time-varying characteristics of the data in different time periods. The last approach (nonadaptive GPD model) does not utilize a sliding window and uses all the available data up to the day on which forecasts are generated. This approach is preferable since

\footnotetext{
16 Danielsson and Moritomo (2000) also adopts a similar windowing approach.
} 

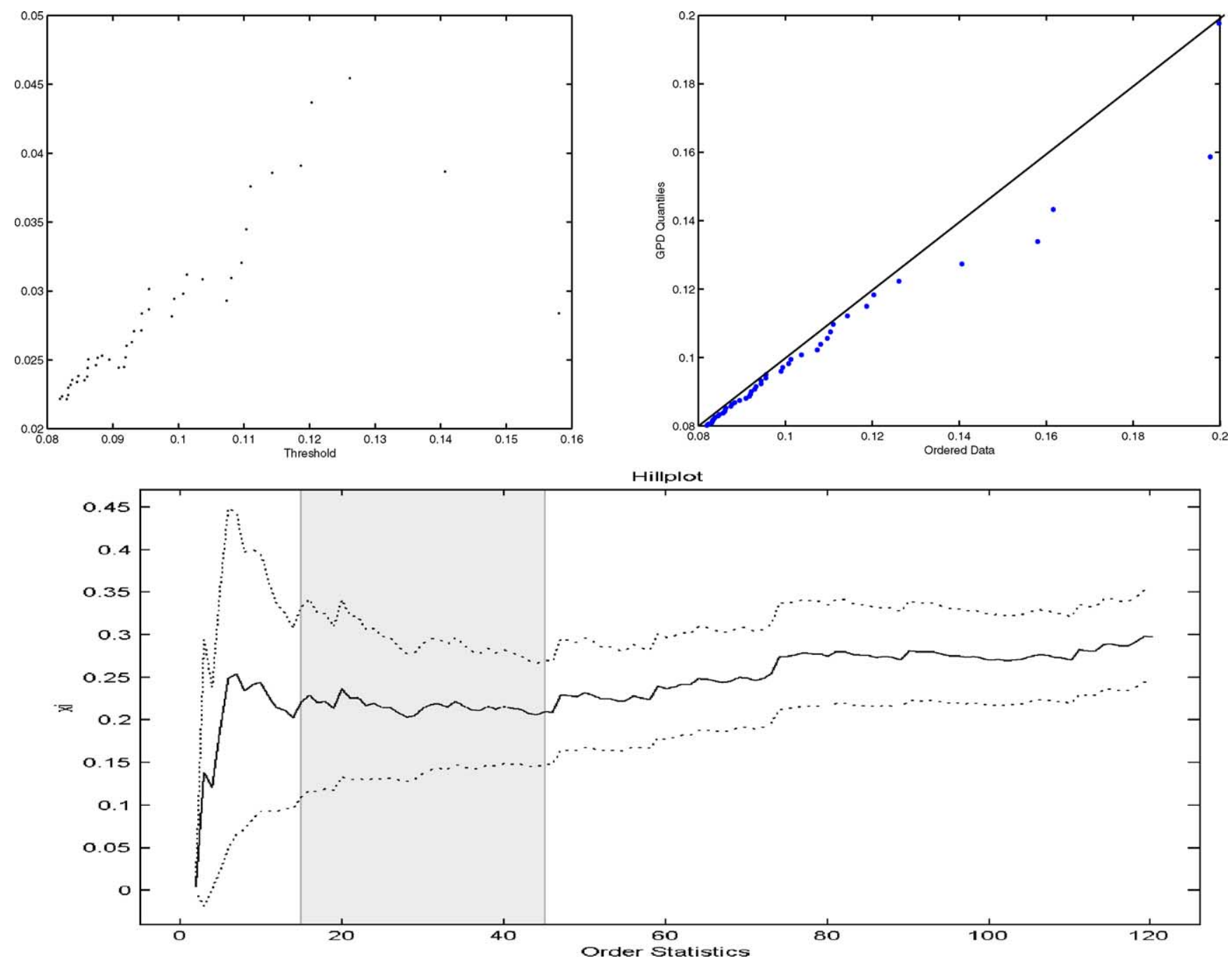

Fig. 3. Top-left: mean excess (ME) plot. The horizontal axis is for thresholds over which the sample mean of the excesses are calculated. Values on the vertical axis display the corresponding mean excesses. The positive trend for thresholds above approximately 0.082 indicates a heavy left tail since this is the mean excess plot for losses. Since an approximately linear positive trend in an ME plot results from a Pareto type behavior (tail probabilities decaying as a power function), the extreme losses in ISE-100 Index have a Pareto type behavior. Top-right: QQ plot of losses with respect to a GPD (assumed shape parameter, $\hat{\xi}$, is 0.20). It shows that the left tail of the distributions over the threshold value 0.08 is well approximated by the GPD. Bottom: variation of the Hill estimate of the shape parameter across the number of upper order statistics. The Hill estimate is very sensitive to the number of upper order statistics. The estimator for the shape parameter should be chosen from a region where the estimate is relatively stable. A stable region is toned gray in the figure. Notice that the confidence bands decrease on the right side of this stability region since more upper order statistics are used to calculate the estimate. 
GPD estimation requires more data for out-of-sample forecasts, as extreme events are rare. ${ }^{17}$

The GARCH models are parameterized as having one autoregressive and one moving average term, $\operatorname{GARCH}(1,1)$, since it is practically impossible to find the best parameterization for each out-of-sample forecast of a given window size. A similar constraint also applies for the GPD modeling, i.e., the difficulty of choosing the appropriate threshold value for each run. Both the adaptive and nonadaptive GPD quantile forecasts are generated using the upper $2.5 \%$ of the sample. In principle, it is possible to choose different thresholds for different quantiles and different window sizes but this would increase the effect of data snooping. ${ }^{18}$ For the historical simulation, piecewise linear interpolation is chosen to make the empirical distribution function one-to-one. ${ }^{19}$

The relative performance of each model is calculated in terms of the violation ratio. A violation occurs when a realized return is greater than the estimated return. The violation ratio is defined as the total number of violations, divided by the total number of one-period forecasts. ${ }^{20}$ If the model is correct, the expected violation ratio is the tail area for each quantile. At $q$ th quantile, the model predictions are expected to be wrong (underpredict the realized return) $\alpha=(1-q)$ percent of the time. For instance, the model is expected to underpredict the realized return $5 \%$ of the time at the 95th quantile. A high violation ratio at each quantile implies that the model excessively underestimates the realized return (=risk). If the violation ratio at the $q$ th quantile is greater than $\alpha$ percent, this implies exces-

\footnotetext{
${ }^{17}$ In extreme value analysis, we employed the EVIM toolbox of Gençay et al. (2003).

18 See Sullivan et al. (2001) for the implications of data snooping in applied studies.

19 There are other interpolation techniques such as nonlinear interpolation or nonparametric interpolation which can also be used.

${ }^{20}$ For example, for a sample size of 3000 , a model with a window size of 500 days produces 2500 one-step-ahead return estimates for a given quantile. Each of these one-step-ahead returns is compared to the corresponding realized return. If the realized return is greater than the estimated return, a violation occurs. The ratio from total violations (total number of times a realized return is greater than the corresponding estimated return) to the total number of estimates is the violation ratio. If the number of violations is 125 , the violation ratio at this particular quantile is $5 \%(125 / 2500=0.05)$. That is, $5 \%$ of the time the model underpredicts the return (realized return is greater than the estimated return).
}

sive underprediction of the realized return. If the violation ratio is less than $\alpha$ percent at the $q$ th quantile, there is excessive overprediction of the realized return by the underlying model. For example, if the violation ratio is $2 \%$ at the 95th quantile, the realized return is only $2 \%$ of the time greater than what the model predicts.

It is tempting to conclude that a small violation ratio is always preferable at a given quantile. However, this may not be the case in this framework. Notice that the estimated return determines how much capital should be allocated for a given portfolio assuming that the investor has a short position in the market. Therefore, a violation ratio excessively greater than the expected ratio implies that the model signals less capital allocation and the portfolio risk is not properly hedged. In other words, the model increases the risk exposure by underpredicting it. On the other hand, a violation ratio excessively lower than the expected ratio implies that the model signals a capital allocation more than necessary. In this case, the portfolio holder allocates more to liquidity and registers an interest rate loss. A regulatory body may prefer a model overpredicting the risk since the institutions will allocate more capital for regulatory purposes. Institutions would prefer a model underpredicting the risk, since they have to allocate less capital for regulatory purposes, if they are using the model only to meet the regulatory requirements. For this reason, the implemented capital allocation ratio is increased by the regulatory bodies for those models that consistently underpredict the risk.

Quantiles which are important for contemporary risk management applications as well as regulatory capital requirements are 0.95 th, 0.975 th, 0.99 th, 0.995 th and 0.999th quantiles. Table 1 displays the violation ratios for the left tail (losses) at the window size of 1000 observations. ${ }^{21}$ The numbers in parentheses are the ranking between six competing models for each quantile. Var-Cov method has the worst performance regardless of the window size except for the 95th quantile. Since quantiles higher than 0.95 th are more of a concern in risk management applications, we can conclude that the Var-Cov method should be placed at the bottom of the performance ranking of competing models in this particular market. The

\footnotetext{
${ }^{21}$ To minimize the space for tables and the corresponding figures we report the results for the window size of 1000 observations. The findings for the window sizes of 500 and 2000 observations do not differ from the window size of 1000 observations significantly.
} 
Table 1

VaR violation ratios for the left tail (losses) of daily ISE-100 returns (in \%) ${ }^{\mathrm{a}}$

\begin{tabular}{|c|c|c|c|c|c|}
\hline & $5 \%$ & $2.5 \%$ & $1 \%$ & $0.5 \%$ & $0.1 \%$ \\
\hline Var-Cov & $5.37(2)$ & $3.36(5)$ & $2.31(6)$ & $1.60(5)$ & $0.92(6)$ \\
\hline Historical simulation & $5.46(3)$ & $2.73(2)$ & $1.22(3)$ & $0.67(1)$ & 0.34 (4) \\
\hline $\operatorname{GARCH}(1,1)$ & 4.70 & $2.85(4)$ & $1.85(5)$ & $1.34(4)$ & $0.59(5)$ \\
\hline GARCH( $(1,1)-t$ & $3.78(6)$ & $2.23(3)$ & $1.18(2)$ & $0.76(3)$ & $0.21(2)$ \\
\hline Adaptive GPD & $6.21(5)$ & $2.73(2)$ & $1.13(1)$ & $0.67(1)$ & $0.25(3)$ \\
\hline Nonadaptive GPD & $4.41(4)$ & $2.64(1)$ & $1.39(4)$ & $0.71(2)$ & $0.17(1)$ \\
\hline
\end{tabular}

${ }^{a}$ The numbers in parentheses are the ranking between six competing models for each quantile. The violation ratio of the best performing model is given in italics. Each model is estimated for a rolling window size of 1000 observations. The expected value of the VaR violation ratio is the corresponding tail size. For example, the expected VaR violation ratio at 5\% tail is 5\%. A calculated value greater than the expected value indicates an excessive underestimation of the risk while a value less than the expected value indicates an excessive overestimation. Daily returns are calculated from the ISE-100 Index. The sample period is 2 November 1987-8 June 2001. The sample size is 3383 observations. Data source: The Central Bank of the Republic of Turkey.

Table 2

VaR violation ratios for the right tail of daily ISE-100 returns (in \%)

\begin{tabular}{|c|c|c|c|c|c|}
\hline & $5 \%$ & $2.5 \%$ & $1 \%$ & $0.5 \%$ & $0.1 \%$ \\
\hline Var-Cov & $4.70(2)$ & $3.19(5)$ & $2.06(6)$ & $1.43(6)$ & $0.59(6)$ \\
\hline Historical simulation & $5.37(3)$ & $2.85(4)$ & $1.34(3)$ & $0.84(4)$ & $0.34(4)$ \\
\hline $\operatorname{GARCH}(1,1)$ & $4.83(1)$ & $2.69(2)$ & $1.47(5)$ & $0.88(5)$ & $0.42(5)$ \\
\hline $\operatorname{GARCH}(1,1)-t$ & $3.74(6)$ & $1.81(5)$ & $0.88(1)$ & $0.42(1)$ & $0.21(2)$ \\
\hline Adaptive GPD & $6.09(4)$ & $2.81(3)$ & $1.22(2)$ & $0.63(2)$ & $0.29(3)$ \\
\hline Nonadaptive GPD & $3.78(5)$ & $2.52(1)$ & $1.43(4)$ & $0.67(3)$ & $0.13(1)$ \\
\hline
\end{tabular}

${ }^{a}$ The numbers in parentheses are the ranking between six competing models for each quantile. Each model is estimated for a rolling window size of 1000 observations. The expected value of the VaR violation ratio is the corresponding tail size. For example, the expected $\mathrm{VaR}$ violation ratio at $5 \%$ tail is $5 \%$. A calculated value greater than the expected value indicates an excessive underestimation of the risk while a value less than the expected value indicates an excessive overestimation. Daily returns are calculated from the ISE-100 Index. The sample period is 2 November 1987-8 June 2001. The sample size is 3383 observations. Data source: The Central Bank of the Republic of Turkey.

second worst model is $\operatorname{GARCH}(1,1)$, except for its excellent performance at the 95th quantile. Although it performs better than the Var-Cov approach, even the simple historical simulation approach produces smaller VaR violation rates than the GARCH model for most quantiles.

At the 0.975th quantile, nonadaptive GPD performs the best with a violation ratio of $2.64 \%$ which amounts to $0.14 \%$ over-rejection. The adaptive GPD models follow with $2.73 \%$ ( $0.23 \%$ over-rejection) and GARCH- $t$ ranks third with $2.23 \% \quad(0.27 \%$ under-rejection). At the 0.99th quantile, the adaptive GPD provides the best violation ratio with $1.13 \%$ which is followed by GARCH- $t$ with $1.18 \%$. At the 0.995 th quantile the adaptive GPD and historical simulation provide the best violation ratios with $0.67 \%$ which is followed by adaptive GPD with $0.71 \%$. At the 0.999th quantile, nonadaptive GPD provides the best performance with $0.17 \%$ which is followed by GARCH- $t$ with $0.21 \%$. Overall, the results in Table 1 indicate that GPD models provide the best violation ratios for quantiles 0.975 and higher. GARCH- $t$ comes close to being the third contender and competes with the historical simulation.

Table 2 displays the results for the right tail of returns. $^{22}$ The Var-Cov method is again the worst model for the quantiles higher than the 0.95th quantile. $\operatorname{GARCH}(1,1)$ performs best at the 0.95th and 0.975 th quantiles but its performance deteriorates at higher quantiles. GARCH $(1,1)-t$ provides the best results for quantiles higher than the 0.975 th except the

\footnotetext{
${ }^{22}$ It is important to investigate both tails since a financial institution may have a short position in the market.
} 
0.999th quantile where nonadaptive GPD performs best. Adaptive GPD is the second best for 0.99th and 0.995 th quantiles. Historical simulation is again an average model.

The one-period ahead 0.99th quantile forecasts of $\operatorname{GARCH}(1,1)$ and $\operatorname{GARCH}(1,1)-t$ models for losses are presented in Fig. 4 for the window size of 1000. Although the daily quantile forecasts of both models are quite volatile, $\operatorname{GARCH}(1,1)-t$ model yields significantly higher, and therefore more volatile quantile forecasts relative to the $\operatorname{GARCH}(1,1)$ model. This implies that the allocation of the capital for regulatory purposes has to vary on a daily basis. This daily variation can be as large as $20 \%$ which is quite costly to implement and difficult to supervise in practice.
In the top panel of Fig. 5 quantile forecasts for the Var-Cov, historical simulation and adaptive GPD models are presented. All three models provide rather stable quantile forecasts across volatile return periods. The Var-Cov and historical simulation quantile forecasts are always lower than the adaptive GPD forecasts with Var-Cov quantile forecasts being the most volatile between these three models.

A comparison between the $\operatorname{GARCH}(1,1)-t$ and adaptive GPD model is presented in the bottom panel of Fig. 5. This comparison indicates that GARCH models yield very volatile quantile estimates when compared to the GPD, historical simulation or Var-Cov approaches. The volatility of the GARCH quantile forecasts are twice as much of the GPD quantile forecasts in a number of dates. Based on
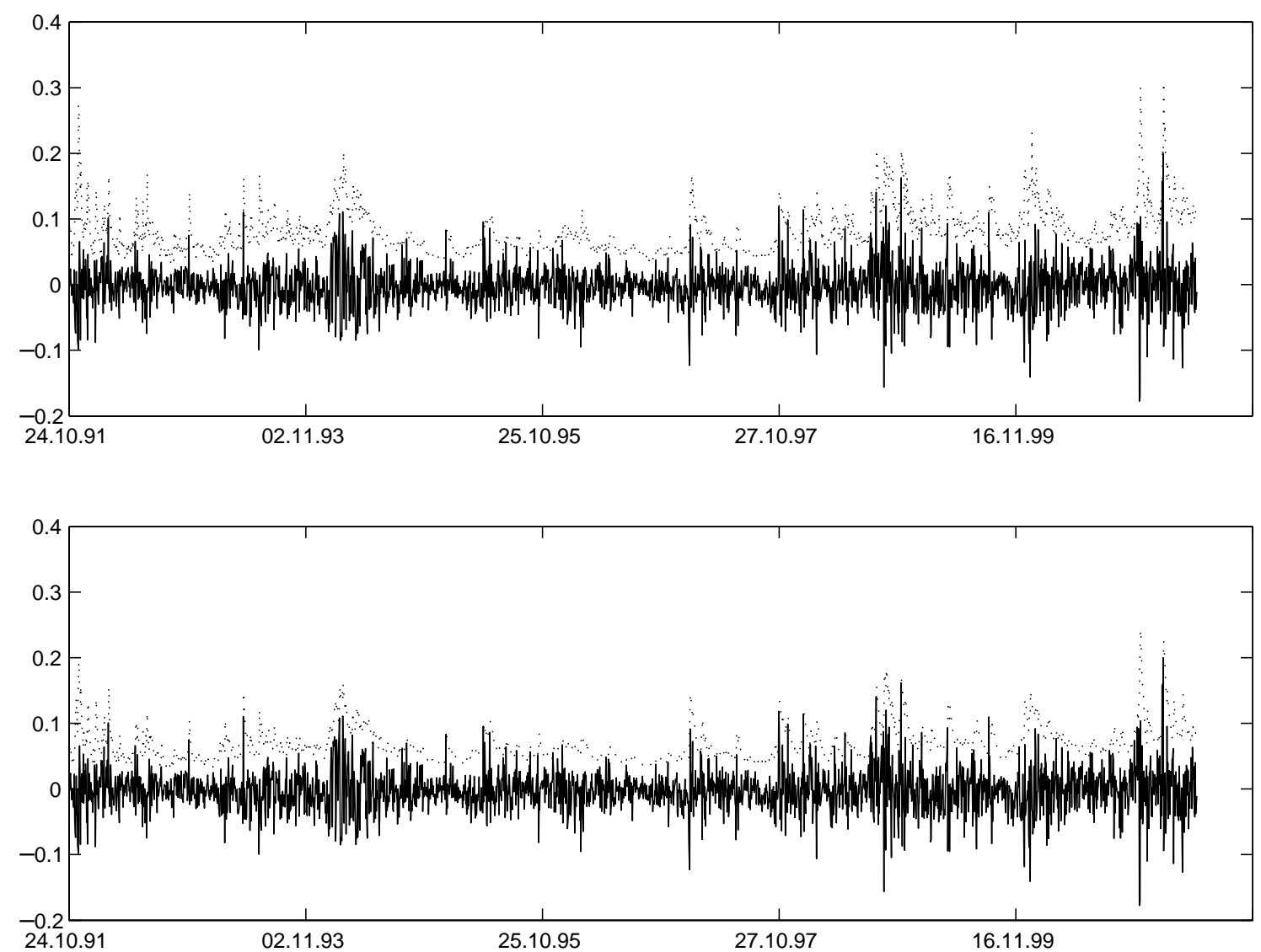

Fig. 4. Daily ISE-100 Index returns from 2 November 1987 to 8 June 2001. Top: one-period ahead 0.99th quantile forecasts (dotted line) of losses (solid line) using a window size of 1000 with $\operatorname{GARCH}(1,1)-t$. Bottom: one-period ahead 0.99th quantile forecasts (dotted line) of losses (solid line) using a window size of 1000 with $\operatorname{GARCH}(1,1)$. Note that for volatile periods, GARCH(1, 1)- $t$ gives significantly higher quantile estimates. 

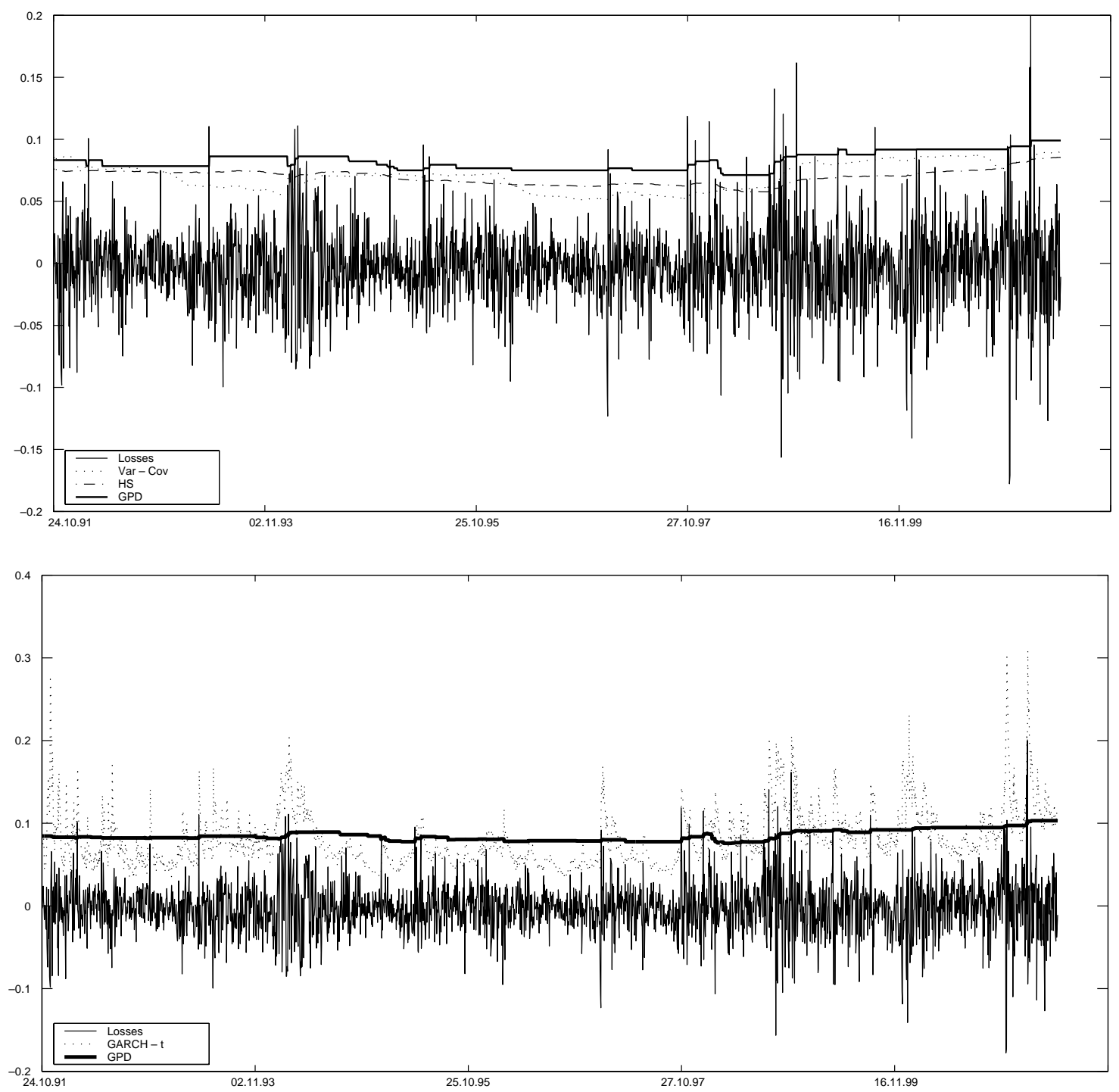

Fig. 5. Daily ISE-100 Index returns from 2 November 1987 to 8 June 2001. Top: one-period ahead 0.99th quantile forecasts of losses using a window size of 1000 with adaptive GPD, historical simulation and Var-Cov methods. The most conservative quantile forecasts belong to the adaptive GPD model. Bottom: one-period ahead 0.99th quantile forecasts of losses using a window size of 1000 with GARCH(1, $1)-t$ and adaptive GPD methods. It is apparent from the figure that $\operatorname{GARCH}(1,1)-t$ quantile forecasts are much more volatile than the adaptive GPD model. Although the $\operatorname{GARCH}(1,1)-t$ model provides more precise forecasts of this quantile, the excessive volatility of the forecasts of the $\operatorname{GARCH}(1,1)-t$ model should be a concern for a risk manager.

Fig. 5, the level of change in the GARCH quantile forecasts can be as large $20-25 \%$ on a daily basis.

It is important that the models to be used in risk management should produce relatively stable quantile forecasts since adjusting the implemented capital frequently (daily) in light of the estimated VaR is costly to implement and regulate. Therefore, models which yield more stable quantile forecasts may be more appropriate for the market risk management purposes. In this respect, the GPD models provide robust tail estimates, and therefore more stable VaR projections in turbulent times. 


\section{3. $S \& P-500$ returns}

Although the Istanbul Stock Exchange Index returns provide an excellent environment to study the VaR models in high volatility markets with thick-tailed distributions, this data set has not been studied widely in the literature and is not well known. Hence, we have repeated the same study with the S\&P-500 Index returns.

The data set is the daily closings of the S\&P-500 Index from 3 January 1983 to 31 December 1996 and there are 3539 observations. The daily returns are defined by $r_{t}=\log \left(p_{t} / p_{t-1}\right)$, where $p_{t}$ denotes the value of the index at day $t$. The top panel of Fig. 6 provides the histogram of the daily S\&P-500 daily returns together with the best fitted normal distribution. The lower panel of Fig. 6 provides the zoomed right tail which indicates thicker tails. The S\&P-500 returns are highly skewed with a sample skewness of -6.5388 and has a large excess kurtosis with a sample kurtosis of 233.79 .

The mean excess plot in the top panel of Fig. 7 indicates a heavy right tail for the loss distribution. The QQ plot in the middle panel of Fig. 7 provides further evidence for fat-tailness. The losses over a threshold are plotted with respect to the GPD with an assumed shape parameter, $\hat{\xi}=0.30$. It clearly shows that the left tail of the distributions over the threshold value 0.08 is well approximated by GPD. The Hill plot is used to calculate the shape parameter $\xi=1 / \alpha$, where $\alpha$ is the tail index. The shape parameter $\xi$ is informative regarding the limiting distribution of maxima. If $\xi=0, \xi>0$ or $\xi<0$, this indicates an exponentially decaying, power-decaying, or finite-tail dis- tributions in the limit, respectively. The Hill plot of losses is displayed in the bottom panel of Fig. 7. The stable portion of this figure implies a tail index estimate of 0.40. Therefore, the Hill estimator indicates a power-decaying tail with an exponent of 2.5.

Table 3 displays the violation ratios for the left tail (losses) at the window size of 1000 observations. The numbers in parentheses are the ranking between six competing models for each quantile. Adaptive GPD model provides the best violation ratio for 0.95 th and 0.975 th quantiles. Var-Cov and historical simulation also do equally well with the adaptive GPD model for the 0.975 th quantile. GARCH- $t$ is the best performer for the 0.99th quantile which is followed by the adaptive GPD model. The nonadaptive GPD model provides the best performance for the 0.995th and 0.999th quantiles where the second best performer is the adaptive GPD model. The results from the left tail analysis indicate that GPD models provide the best violation ratios in most quantiles. The ranking amongst the remaining three models is not obvious although Var-Cov method receives the worst violation ratios at the 0.99th quantiles and higher. Table 4 displays the results for the right tail of returns. Amongst five quantiles, the adaptive GPD model performs as the best model by ranking first in three quantiles and the second in the remaining two quantiles. GARCH- $t$ model has the worst performance in this tail by ranking as the last model except at the 0.999th quantile.

The S\&P-500 one-period ahead 0.99th quantile forecasts of $\operatorname{GARCH}(1,1)$ and $\operatorname{GARCH}(1,1)-t$ models for negative returns (losses) are presented in Fig. 8 for the window size of 1000 . Although the daily quantile forecasts of both models are quite volatile,

Table 3

VaR violation ratios for the left tail (losses) of daily S\&P-500 returns (in \%) ${ }^{\mathrm{a}}$

\begin{tabular}{|c|c|c|c|c|c|}
\hline & $5 \%$ & $2.5 \%$ & $1 \%$ & $0.5 \%$ & $0.1 \%$ \\
\hline Var-Cov & $3.39(4)$ & 2.40 & $1.69(5)$ & $1.42(5)$ & 0.75 \\
\hline Historical simulation & $4.61(3)$ & 2.60 & $1.50(4)$ & $0.75(3)$ & $0.20(2)$ \\
\hline $\operatorname{GARCH}(1,1)$ & $4.71(2)$ & 2.89 (3) & $2.18(6)$ & $1.58(6)$ & $0.83(4)$ \\
\hline $\operatorname{GARCH}(1,1)-t$ & $3.29(6)$ & $1.98(4)$ & 1.07 (1) & $0.83(4)$ & $0.31(3)$ \\
\hline Adaptive GPD & $4.72(1)$ & 2.60 & $1.30(3)$ & $0.63(2)$ & $0.12(1)$ \\
\hline Nonadaptive GPD & $3.19(5)$ & $2.20(2)$ & $1.10(2)$ & $0.47(1)$ & $0.12(1)$ \\
\hline
\end{tabular}

a The numbers in parentheses are the ranking between six competing models for each quantile. Each model is estimated for a rolling window size of 1000 observations. The expected value of the VaR violation ratio is the corresponding tail size. For example, the expected $\mathrm{VaR}$ violation ratio at $5 \%$ tail is $5 \%$. A calculated value greater than the expected value indicates an excessive underestimation of the risk while a value less than the expected value indicates an excessive overestimation. Daily returns are calculated from the S\&P-500 Index. The sample period is 3 January 1983-31 December 1996. The sample size is 3539 observations. Data source: Datastream. 

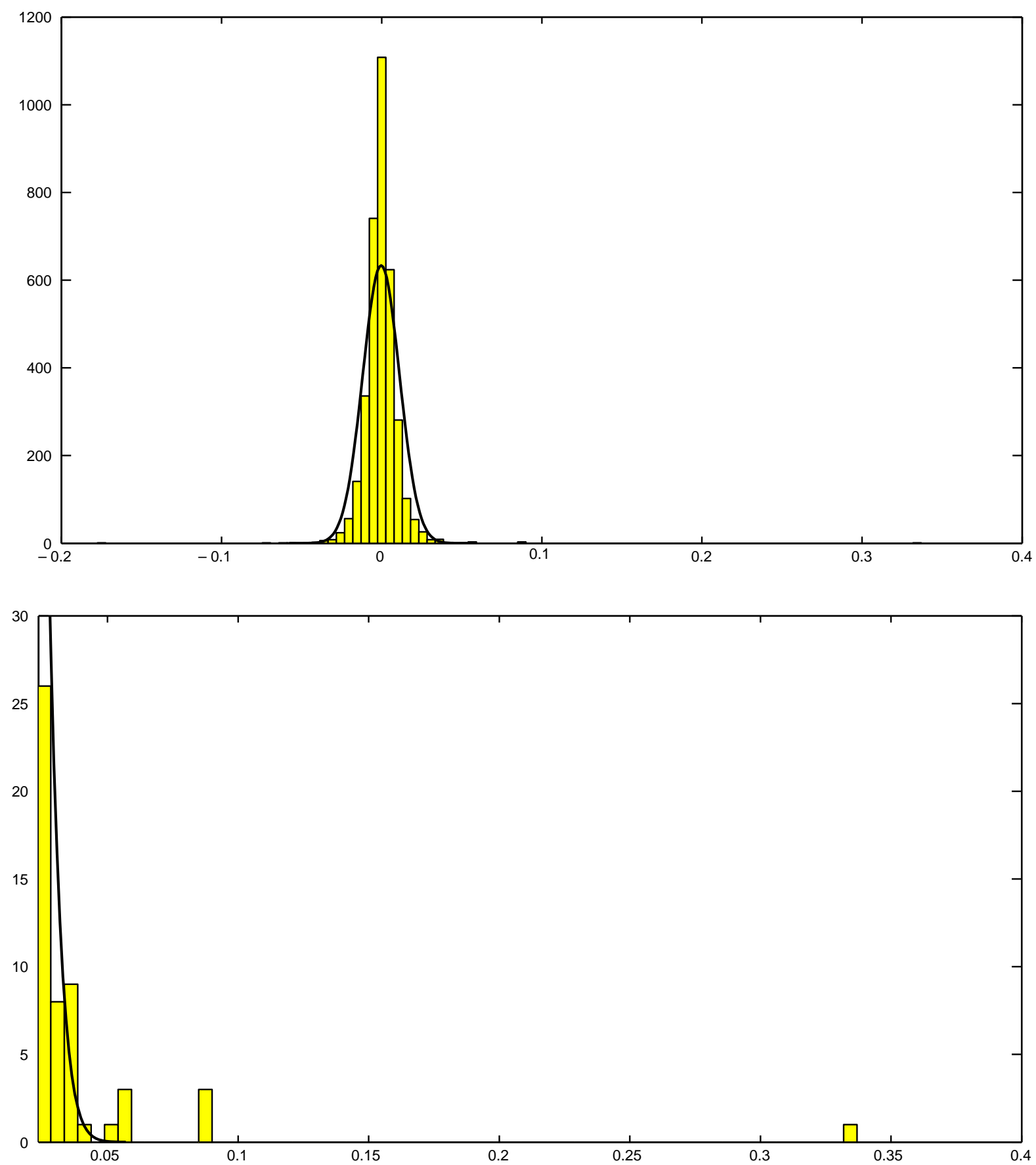

Fig. 6. Histogram of S\&P-500 daily negative returns (losses) and the best fitted normal distribution, $N(\hat{\mu}, \hat{\sigma})$. Estimated parameters are $\hat{\mu}=-0.0003$ and $\hat{\sigma}=0.0115$. The right tail region (values over $\hat{\mu}+2 \hat{\sigma}=0.0227$ ) is zoomed in the lower panel. The sample period is 3 January 1983 to 31 December 1996. The sample size is 3539 observations. 

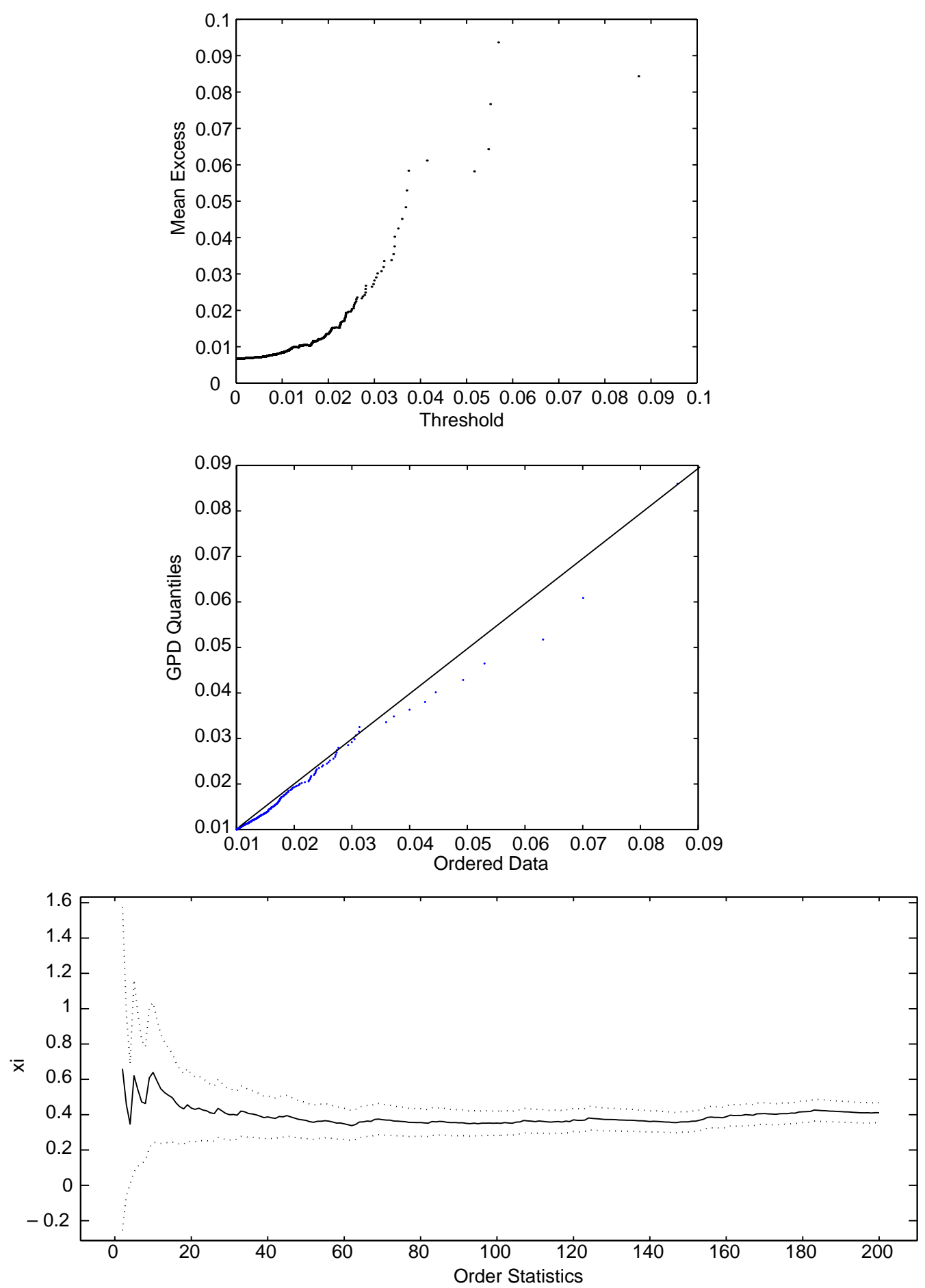

Fig. 7. Top: ME plot; middle: QQ plot; bottom: Hill plot of the S\&P-500 returns. The sample period is 3 January 1983 to 31 December 1996. The sample size is 3539 observations. 
Table 4

VaR violation ratios for the right tail of daily S\&P-500 returns (in \%)

\begin{tabular}{|c|c|c|c|c|c|}
\hline & $5 \%$ & $2.5 \%$ & $1 \%$ & $0.5 \%$ & $0.1 \%$ \\
\hline Var-Cov & 3.03 (4) & 1.97 (2) & $1.06(2)$ & 0.75 (4) & $0.31(5)$ \\
\hline Historical simulation & $4.57(1)$ & $2.52(1)$ & $1.06(2)$ & $0.63(3)$ & $0.16(3)$ \\
\hline $\operatorname{GARCH}(1,1)$ & $3.31(3)$ & $1.91(3)$ & $1.01(1)$ & $0.79(5)$ & $0.22(4)$ \\
\hline $\operatorname{GARCH}(1,1)-t$ & $1.80(6)$ & $0.84(5)$ & $0.22(4)$ & $0.11(6)$ & $0.06(2)$ \\
\hline Adaptive GPD & $5.75(2)$ & $2.52(1)$ & $0.99(1)$ & $0.55(2)$ & $0.12(1)$ \\
\hline Nonadaptive GPD & $2.72(5)$ & $1.61(4)$ & $0.83(3)$ & $0.47(1)$ & $0.12(1)$ \\
\hline
\end{tabular}

${ }^{a}$ The numbers in parentheses are the ranking between six competing models for each quantile. Each model is estimated for a rolling window size of 1000 observations. The expected value of the VaR violation ratio is the corresponding tail size. For example, the expected VaR violation ratio at $5 \%$ tail is $5 \%$. A calculated value greater than the expected value indicates an excessive underestimation of the risk while a value less than the expected value indicates an excessive overestimation. Daily returns are calculated from the S\&P-500 Index. The sample period is 3 January 1983-31 December 1996. The sample size is 3539 observations. Data source: Datastream.
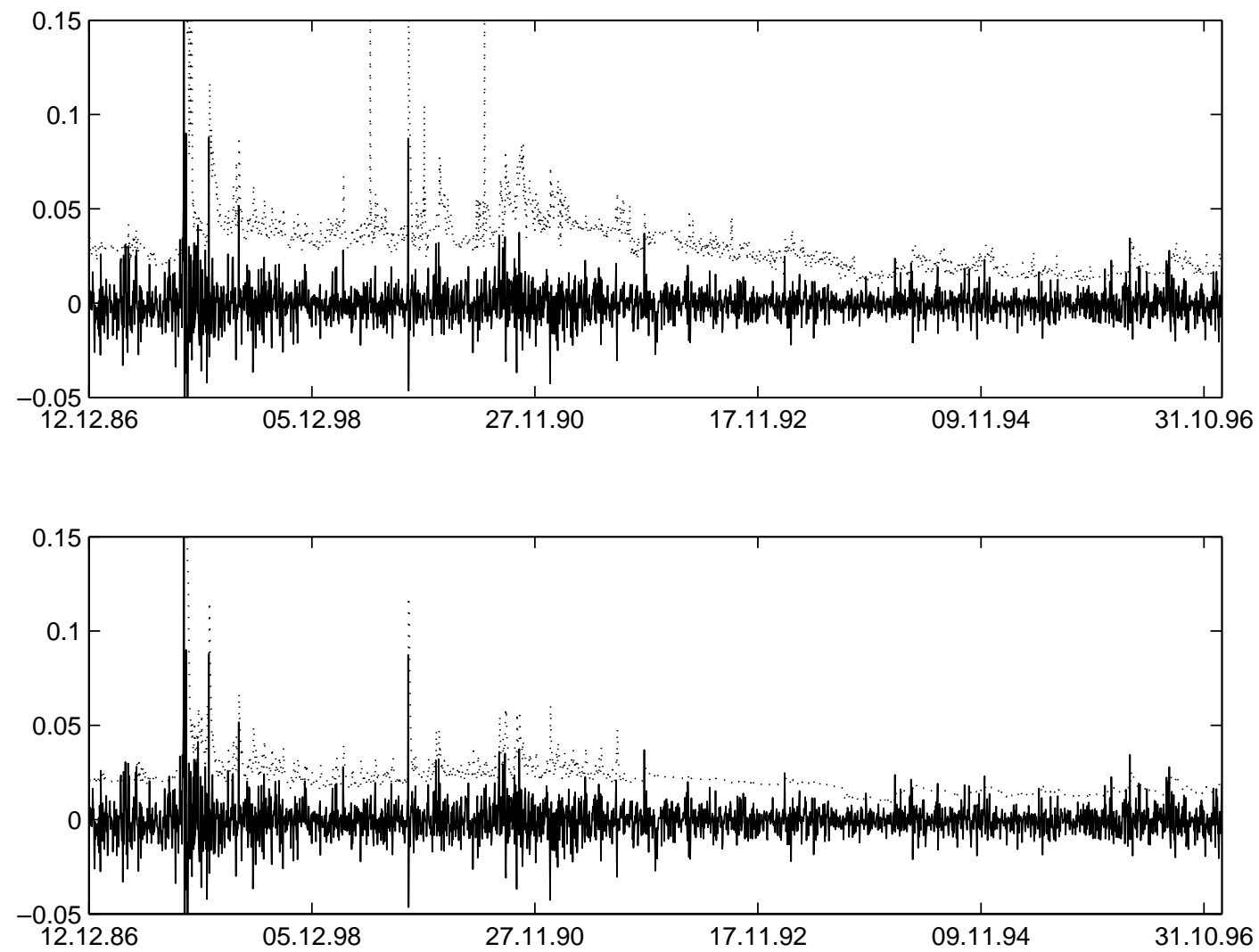

Fig. 8. Top: S\&P-500 one-period ahead 0.99th quantile forecasts (dotted line) of losses (solid line) using a window size of 1000 with $\operatorname{GARCH}(1,1)-t$. Bottom: one-period ahead 0.99th quantile forecasts (dotted line) of losses (solid line) using a window size of 1000 with $\operatorname{GARCH}(1,1)$. Note that for volatile periods $\operatorname{GARCH}(1,1)-t$ gives significantly higher quantile estimates. We restricted the vertical axis to $[-0.05,0.15]$ to improve the resolution. Otherwise $\mathrm{GARCH}$ quantile forecasts are as large as 0.34 . 
$\operatorname{GARCH}(1,1)-t$ model yields significantly higher, and therefore more volatile quantile forecasts relative to the $\operatorname{GARCH}(1,1)$ model. This implies that the allocation of the capital for regulatory purposes has to vary on a daily basis. This daily variation can be as large as
$30 \%$ which is quite costly to implement and difficult to supervise in practice. The findings from the left tail are parallel to the ones from the ISE-100 analysis. In the top panel of Fig. 9 quantile forecasts for the Var-Cov, historical simulation and adaptive GPD
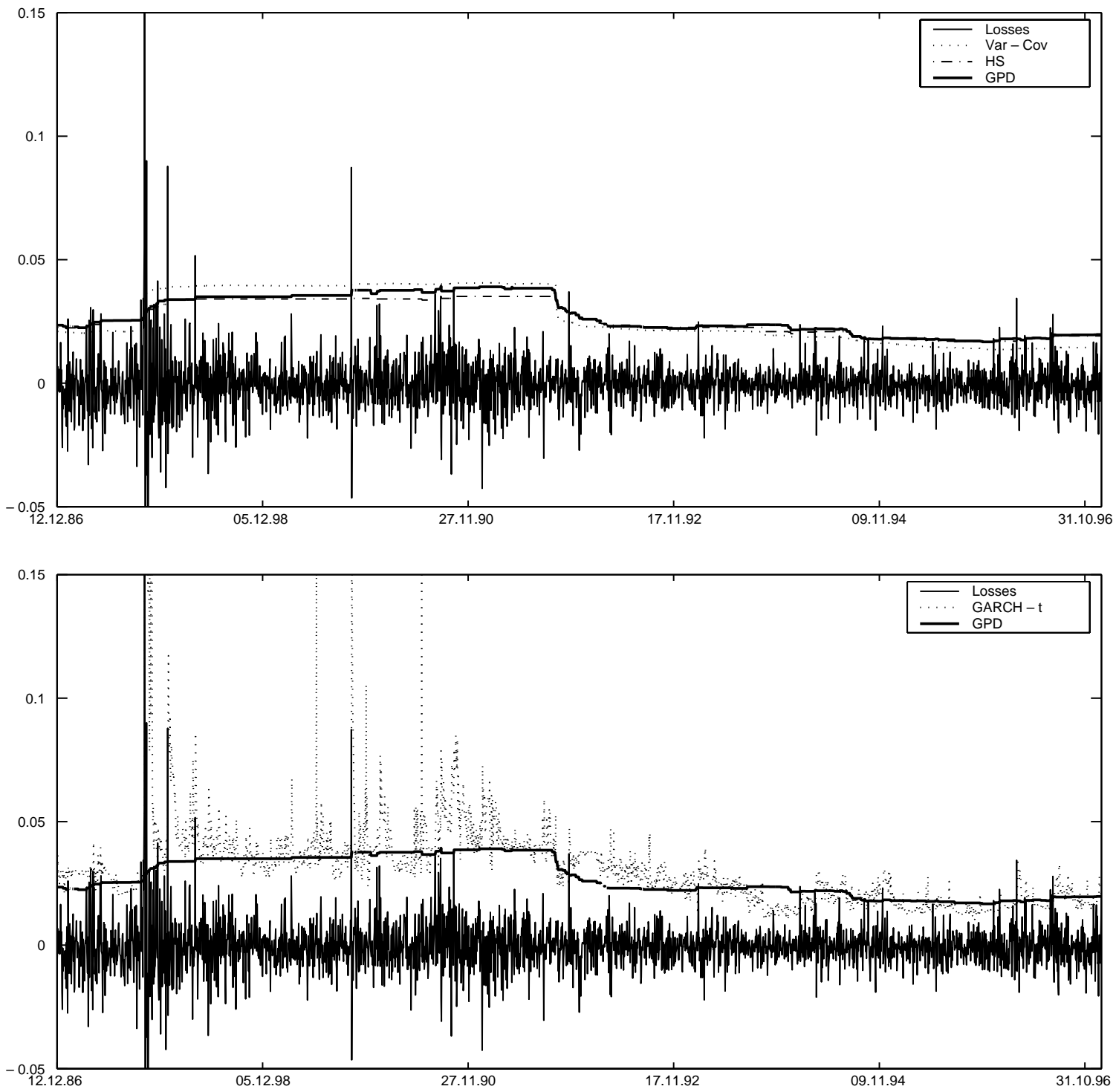

Fig. 9. Top: S\&P-500 one-period ahead 0.99th quantile forecasts of losses using a window size of 1000 with adaptive GPD, historical simulation and Var-Cov methods. The most conservative quantile forecasts belong to the adaptive GPD model. Bottom: one-period ahead 0.99th quantile forecasts of losses using a window size of 1000 with $\operatorname{GARCH}(1,1)$ - $t$ and adaptive GPD methods. It is apparent from the figure that $\operatorname{GARCH}(1,1)-t$ quantile forecasts are much more volatile than the adaptive GPD model. Although the GARCH(1, 1)- $t$ model provides more precise forecasts of this quantile, the excessive volatility of the forecasts of the GARCH(1, 1)- $t$ model should be a concern for a risk manager. We restricted the vertical axis to $[-0.05,0.15]$ to improve the resolution. Otherwise GARCH quantile forecasts are as large as 0.34 . 
models are presented. All three models provide rather stable quantile forecasts across volatile return periods. The Var-Cov and historical simulation quantile forecasts follow similar time paths as the adaptive GPD quantile forecasts.

A comparison between the $\operatorname{GARCH}(1,1)-t$ and adaptive GPD model is presented in the bottom panel of Fig. 9. This comparison indicates that GARCH models yield very volatile quantile estimates when compared to the GPD, historical simulation or Var-Cov approaches. The volatility of the GARCH quantile forecasts are multiples of the GPD quantile forecasts in a number of dates. Based on Fig. 9, the level of change in the GARCH quantile forecasts can be as large as $30 \%$ on a daily basis. Our findings from the S\&P-500 returns confirm the findings obtained from the ISE-100 returns that the GPD model provides more accurate violation ratios and its quantile forecasts are stable across turbulent times.

From a regulatory point of view, it is important that banks maintain enough capital to protect themselves against extreme market conditions. This concern allows regulators to impose minimum capital requirements in different countries. In 1996, the Basel Committee recommended a framework for measuring market risk and credit risk, and for determining the corresponding capital requirements, see Basel (1996). ${ }^{23}$ The committee proposes two different ways of calculating the minimum capital risk requirement: a standardized approach and an internal risk management model. In the standardized approach, banks are required to slot their exposures into different supervisory categories. These categories have fixed risk weights set by the regulatory authorities. A bank can utilize its own internal risk management model, subject to approval by the authorities. These models must meet certain conditions. Our "violation ratio" above as a criterion for evaluation of different models is basically the Basel Committee criterion for evaluating internal risk management models. We showed that this criterion, in combination with volatile mar-

\footnotetext{
23 The New Basel Capital Accord (Basel II) is expected to be finalized by the end of 2003 with implementation to take place in member countries by year-end 2006. According to a recent consultative document by Basel Committee, the new accord substantially changes the treatment of credit risk and introduces an explicit treatment operational risk; see http://www.bis.org for further details.
}

ket conditions, may result in costly implementations, especially if conditional models are employed to measure the risk. The results also indicate that the existing Basel Committee risk measurement and regulatory framework can be improved by incorporating costs of trading, costs of capital adjustments and the amount of losses into the existing criterion to determine minimum capital requirements.

\section{Conclusions}

Risk management gained importance in the last decade due to the increase in the volatility of financial markets and a desire of a less fragile financial system. In risk management, the VaR methodology as a measure of market risk is popular with both financial institutions and regulators. VaR methodology benefits from the quality of quantile forecasts. In this study, conventional models such as GARCH, historical simulation and Var-Cov approaches, are compared to EVT models. The six models used in this study can be classified into two groups: one group consisting of $\operatorname{GARCH}(1,1)$ and $\operatorname{GARCH}(1,1)-t$ models which lead to highly volatile quantile forecasts, while the other group consisting of historical simulation, Var-Cov, adaptive GPD and nonadaptive GPD models provide more stable quantile forecasts. In the first group, $\operatorname{GARCH}(1,1)-t$, while in the second group the GPD model is preferable for most quantiles.

Our results suggest further study by constructing a cost function that penalizes the excessive volatility and rewards the accuracy of the quantile forecasts at the same time. The results also indicate that the existing Basel committee risk measurement and regulatory framework can be improved by incorporating costs of trading, costs of capital adjustments and the amount of losses into existing criterion to determine minimum capital requirements.

\section{Acknowledgements}

Ramazan Gençay gratefully acknowledges financial support from the Swiss National Science Foundation under NCCR-FINRISK, Natural Sciences and Engineering Research Council of Canada and the Social Sciences and Humanities Research Council of Canada. 


\section{References}

Basel, 1996. Overview of the Amendment to the Capital Accord to Incorporate Market Risk. Basel Committee on Banking Supervision, Basel.

Bera, A., Jarque, C., 1981. Efficient tests for normality, heteroscedasticity and serial independence of regression residuals: Monte Carlo evidence. Economics Letter 7, 313-318.

Bollerslev, T., 1982. Generalized autoregressive conditional heteroscedasticity. Journal of Econometrics 31, 307-327.

Boothe, P., Glassman, P.D., 1987. The statistical distribution of exchange rates. Journal of International Economics 22, 297319.

Dacorogna, M.M., Pictet, O.V., Müller, U.A., de Vries, C.G., 2001a. Extremal forex returns in extremely large datasets. Extremes 4, 105-127.

Dacorogna, M.M., Gençay, R., Müller, U.A., Olsen, R.B., Pictet, O.V., 2001b. An Introduction to High-frequency Finance. Academic Press, San Diego, CA.

Danielsson, J., de Vries, C.G., 1997. Tail index and quantile estimation with very high frequency data. Journal of Empirical Finance 4, 241-257.

Danielsson, J., de Vries, C.G., 2000. Value-at-risk and extreme returns. Annales D'Economie et de Statistique 60, 239-270.

Danielsson, J., Moritomo, J., 2000. Forecasting extreme financial risk: a critical analysis of practical methods for the Japanese market. Monetary Economic Studies 12, 25-48.

de Haan, L., Jansen, D.W., Koedijk, K.G., de Vries, C.G., 1994. Safety first portfolio selection, extreme value theory and long run asset risks. In: Galambos, J., Lechner, J., Kluwer, E.S. (Eds.), Extreme Value Theory and Applications. Kluwer Academic Publishers, Dordrecht, pp. 471-488.

Dowd, K., 1998. Beyond Value-at-Risk: The New Science of Risk Management. Wiley, Chichester, UK.

Duffie, D., Pan, J., 1997. An overview of value-at-risk. Journal of Derivatives 7, 7-49.

Embrechts, P., 1999. Extreme value theory in finance and insurance. Department of Mathematics, ETH, Swiss Federal Technical University.

Embrechts, P., 2000a. Extreme value theory: potentials and limitations as an integrated risk management tool. Derivatives Use, Trading and Regulation 6, 449-456.

Embrechts, P., 2000b. Extremes and Integrated Risk Management. Risk Books and UBS Warburg, London.

Embrechts, P., Kluppelberg, C., Mikosch, C., 1997. Modeling Extremal Events for Insurance and Finance. Springer, Berlin.

Embrechts, P., Resnick, S., Samorodnitsky, G., 1999. Extreme value theory as a risk management tool. North American Actuarial Journal 3, 30-41.

Engle, R.F., 1982. Autoregressive conditional heteroscedastic models with estimates of the variance of United Kingdom inflation. Econometrica 50, 987-1007.

Ertuğrul, A., Selçuk, F., 2001. A brief account of the Turkish economy: 1980-2000. Russian East European Finance Trade 37, 6-28.

Gençay, R., Selçuk, F., 2001. Overnight borrowing, interest rates and extreme value theory. Department of Economics, Bilkent University.
Gençay, R., Selçuk, F., Ulugülyağcı, A., 2003. EVIM: a software package for extreme value analysis in Matlab. Studies in Nonlinear Dynamics and Econometrics 5, 213-239.

Ghose, D., Kroner, K.F., 1995. The relationship between GARCH and symmetric stable distributions: finding the source of fat tails in the financial data. Journal of Empirical Finance 2, 225251.

Hauksson, H.A., Dacorogna, M., Domenig, T., Müller, U., Samorodnitsky, G., 2001. Multivariate extremes, aggregation and risk estimation. Quantitative Finance 1, 79-95.

Hols, M.C., de Vries, C.G., 1991. The limiting distribution of extremal exchange rate returns. Journal of Applied Econometrics 6, 287-302.

Jorion, P., 1997. Value-at-Risk: The New Benchmark for Controlling Market Risk. McGraw-Hill, Chicago.

Koedijk, K.G., Schafgans, M.M.A., de Vries, C.G., 1990. The tail index of exchange rate returns. Journal of International Economics 29, 93-108.

Levich, R.M., 1985. Empirical studies of exchange rates: price behavior, rate determination and market efficiency. Handbook of Economics 6, 287-302.

Loretan, M., Phillips, P.C.B., 1994. Testing the covariance stationary of heavy-tailed time series. Journal of Empirical Finance 1, 211-248.

Lux, T., 1996. The stable Paretian hypothesis and the frequency of large returns: an examination of major German stocks. Applied Financial Economics 6, 463-475.

Mandelbrot, B., 1963a. New methods in statistical economics. Journal of Political Economy 71, 421-440.

Mandelbrot, B., 1963b. The variation of certain speculative prices. Journal of Business 36, 394-419.

Mantegna, R.N., Stanley, H.E., 1995. Scaling behavior in the dynamics of an economic index. Nature 376, 46-49.

McNeil, A.J., 1997. Estimating the tails of loss severity distributions using extreme value theory. ASTIN Bulletin 27, 1117-1137.

McNeil, A.J., 1998. Calculating quantile risk measures for financial time series using extreme value theory. Department of Mathematics, ETH, Swiss Federal Technical University, ETH E-Collection. http://e-collection.ethbib.ethz.ch/.

McNeil, A.J., 1999. Extreme value theory for risk managers. Internal Modeling CAD II, Risk Books, pp. 93-113.

McNeil, A.J., Frey, R., 2000. Estimation of tail-related risk measures for heteroscedastic financial time series: an extreme value approach. Journal of Empirical Finance 7, 271-300.

Müller, U.A., Dacorogna, M.M., Pictet, O.V., 1998. Heavy tails in high-frequency financial data. In: Adler, R.J., Feldman, R.E., Taqqu, M.S. (Eds.), A Practical Guide to Heavy Tails: Statistical Techniques for Analysing Heavy Tailed Distributions. Birkhäuser, Boston, pp. 55-77.

Mussa, M., 1979. Empirical regularities in the behavior of exchange rates and theories of the foreign exchange market. In: Carnegie-Rochester Conference Series on Public Policy, pp. 9-57.

Nelson, D.B., 1991. Conditional heteroskedasticity in asset returns: a new approach. Econometrica 59, 347-370.

Pictet, O.V., Dacorogna, M.M., Müller, U.A., 1998. Hill, bootstrap and jackknife estimators for heavy tails. In: Taqqu, M.S. 
(Ed.), A Practical Guide to Heavy Tails: Statistical Techniques for Analysing Heavy Tailed Distributions. Birkhäuser, Boston, pp. 283-310.

Reiss, R., Thomas, M., 1997. Statistical Analysis of Extreme Values. Birkhäuser, Basel.
Sullivan, R., Timmermann, A., White, H., 2001. Dangers of data-driven inference: the case of calendar effects in stock returns. Journal of Econometrics 105, 249-286.

Teugels, J.B.J., Vynckier, P., 1996. Practical Analysis of Extreme Values. Leuven University Press, Leuven. 